\title{
Mean Reversion versus Random Walk in Oil and Natural Gas Prices
}

\author{
Hélyette Geman \\ Birkbeck, University of London, United Kingdom \\ \& ESSEC Business School, Cergy-Pontoise, France \\ hgeman@ems.bbk.ac.uk
}

Summary. The goals of the paper are as follows: i) review some qualitative properties of oil and gas prices in the last 15 years; ii) propose some mathematical elements towards a definition of mean reversion that would not be reduced to the form of the drift in a stochastic differential equation; iii) conduct econometric tests in order to conclude whether mean reversion still exists in the energy commodity price behavior. Regarding the third point, a clear "break" in the properties of oil and natural gas prices and volatility can be exhibited in the period 2000-2001.

Key words: Oil and gas markets; mean reversion; invariant measure.

\section{Introduction}

Energy commodity prices have been rising at an unprecedented pace over the last five years. As depicted in Figure 1, an investment of $\$ 100$ made in January 2002 in the global Dow Jones-AIG Commodity Index had more than doubled by July 2006, whereas Figure 2 indicates that an investment of $\$ 100$ in the Dow Jones-AIG Energy sub-index had turned into \$500 in July 2005. Among the numerous explanations for this phenomenon, we may identify the severe tensions on oil and their implications for other fossil fuels that may be substitutes. The increase of oil prices is driven by demand growth, particularly in Asia where Chinese consumption rose by 900,000 barrels per day, mostly accounted for by imports.

At the world level, the issue of "peak oil" - the date at which half of the reserves existing at the beginning of time are (will be) consumed - is the subject of intense debates. Matthew Simmons asks in his book, Twilight in the Desert, whether there is a significant amount of oil left in the soil of Saudi Arabia. The concern of depleting reserves in the context of an exhaustive commodity such as oil is certainly present on market participants' minds, and in turn, on the trajectory depicted in Figure 2. 


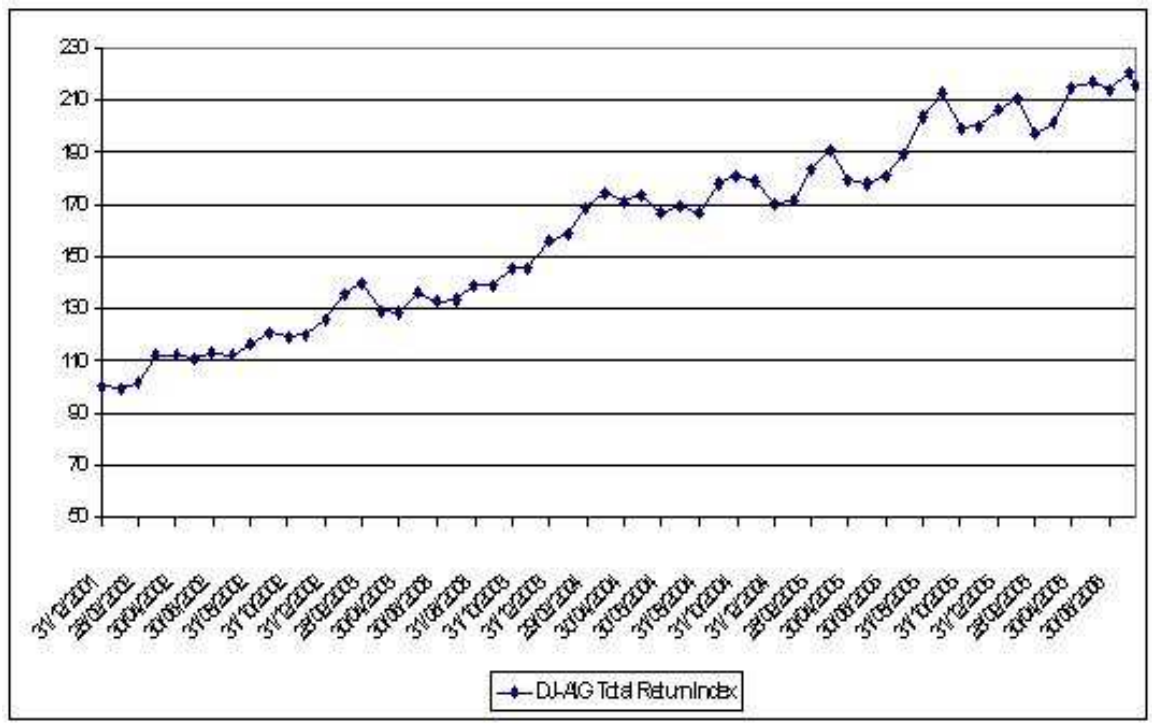

Fig. 1. Dow Jones-AIG Total Return Index over the period Jan 2002-July 2006.

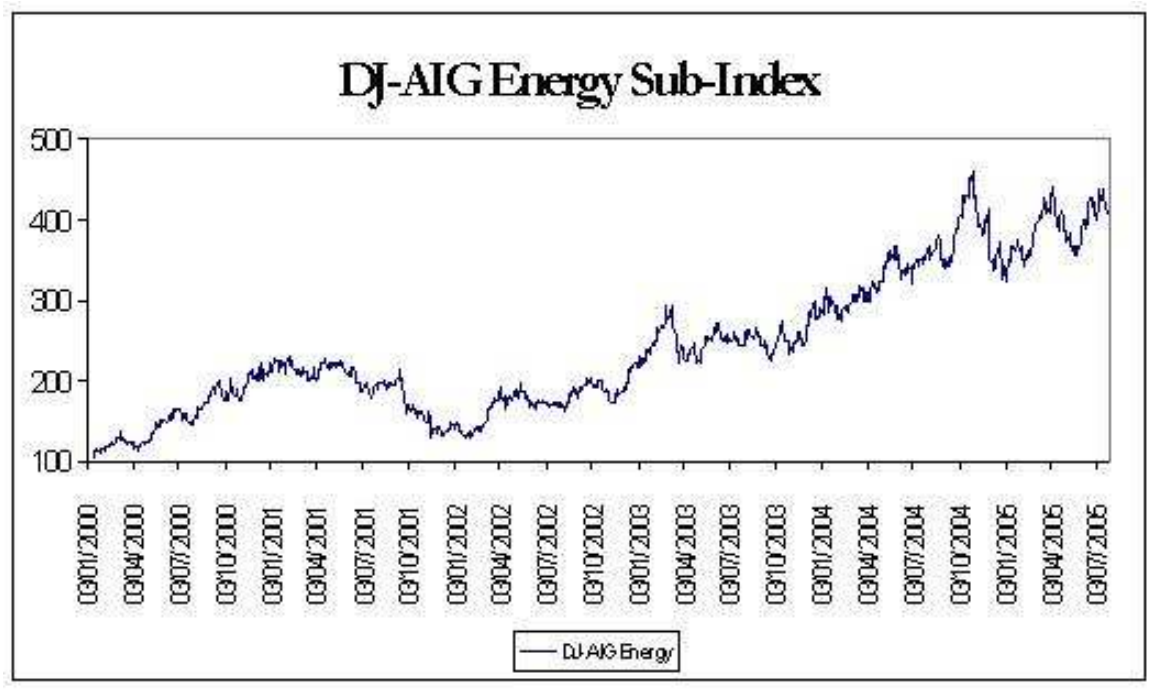

Fig. 2. Dow Jones-AIG Energy Sub-Index over the period Jan 2000-July 2005. 


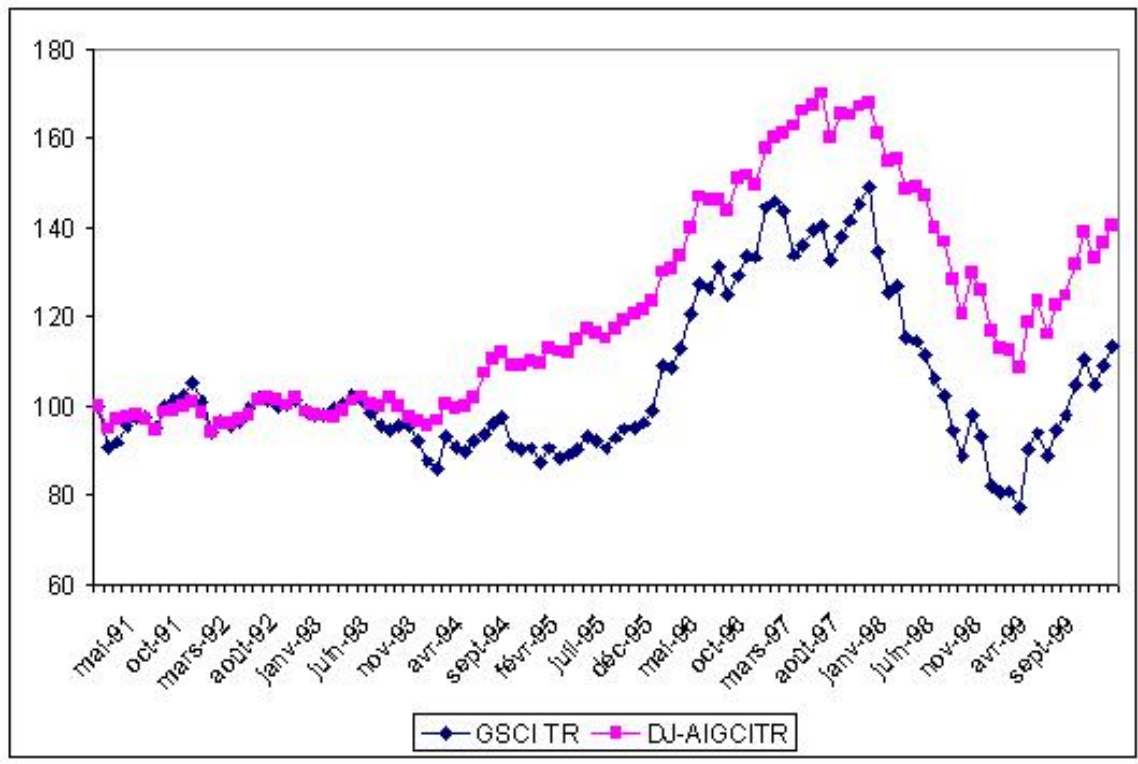

Fig. 3. Goldman Sachs Commodity Index Total Return and Dow Jones-AIG Commodity Index total return over the period Feb 1991-Dec 1999.

The financial literature on commodity price modeling started with the pioneering paper by Gibson and Schwartz [7]. In the spirit of the Black-ScholesMerton [2] formula, they use a geometric Brownian motion for oil spot prices. Given the behavior of commodity prices during the 1990s depicted in Figure 3 by the two major commodity indexes, [10] introduces a mean-reverting drift in the stochastic differential equation driving oil price dynamics; [14], [4], [12] keep this mean-reversion representation for oil, electricity and bituminous coal.

The goal of this paper is to revisit the modelling of oil and natural gas prices in the light of the trajectories observed in the recent past (see Figure 2 ), as well as the definition of mean reversion from a general mathematical perspective. Note that this issue matters also for key quantities in finance such as stochastic volatility. Fouque, Papanicolaou and Sircar [5] are interested in the property of clustering exhibited by the volatility of asset prices. Their view is that volatility is 'bursty' in nature, and burstiness is closely related to mean reversion, since a bursty process is returning to its mean (at a speed that depends on the length of the burst period). 


\section{Some Elements on Mean Reversion in Diffusion from a Mathematical Perspective}

The long-term behavior of continuous time Markov processes has been the subject of much attention, starting with the work of Has'minskii [8]. Accordingly, the long-term evolution of the price of an exhaustible commodity, such as oil or copper, is a topic of major concern in finance, given the world geopolitical and economic consequences of this issue. In what follows, $\left(X_{t}\right)$ will essentially have the economic interpretation of a log-price. We start with a process $\left(X_{t}\right)$ defined as solution of a stochastic differential equation

$$
d X_{t}=b\left(X_{t}\right) d t+\sigma\left(X_{t}\right) d W_{t},
$$

where $\left(W_{t}\right)$ is a standard Brownian motion on a probability space $(\Omega, \mathcal{F}, \mathcal{P})$ describing the randomness of the economy. We know from Itô that if $b$ and $\sigma$ are Lipschitz, there exists a unique solution to the equation. If only $b$ is Lipschitz and $\sigma$ Holder of coefficient $\frac{1}{2}$, we still have existence and uniqueness of the solution. In both cases, the process $\left(X_{t}\right)$ will be Markov and the drift $b\left(X_{t}\right)$ will contain the representation of the trend perceived at date $t$ for future spot prices.

Given a process $\left(X_{t}\right)$, we are in finance particularly interested in the possible existence of a distribution for $X_{0}$ such that, for any positive $t, X_{t}$ has the same distribution. This distribution, if it exists, may be viewed as an equilibrium state for the process. We now recall the definition of an invariant probability measure for a Markov process $\left(X_{t}\right)_{t>0}$, whose semi-group is denoted $\left(P_{t}\right)_{t>0}$ and satisfies the property that, for any bounded measurable function, $P_{t} f(x)=E\left[f\left(X_{t}\right)\right]$.

Definition 1. (i) A measure $\mu$ is said to be invariant for the process $\left(X_{t}\right)$ if and only if

$$
\int \mu(d x) P_{t} f(x)=\int \mu(d x) f(x),
$$

for any bounded function $f$. (ii) $\mu$ is invariant for $\left(X_{t}\right)$ if and only if $\mu P_{t}=\mu$. Equivalently, the law of $\left(X_{t+u}\right)_{u \geq 0}$ is independent of $t$ if we start at date 0 with the measure $\mu$.

Proposition 1. The existence of an invariant measure implies that the process $\left(X_{t}\right)$ is stationary. If $\left(X_{t}\right)$ admits a limit law independent of its initial state, then this limit law is an invariant measure.

Proof. The first part of the proposition is nothing but one of the two forms of the definition above. Now suppose $E\left[f\left(X_{t}\right)\right] \rightarrow_{t \rightarrow \infty} \int \mu(d y) f(y)$, for any bounded function $f$. Then

$$
E_{X}\left[f\left(X_{t+s}\right)\right]=E_{X}\left[P_{S} f\left(X_{t}\right)\right] \rightarrow_{t \rightarrow \infty} \int \mu(d y) f(y) .
$$

Hence, $\mu P_{S}=\mu$, and $\mu$ is invariant. 
Proposition 2. 1. The Ornstein-Uhlenbeck process admits a finite invariant measure, and this measure is Gaussian.

2. The Cox-Ingersoll-Ross (or square-root) process also has a finite invariant measure.

3. The arithmetic Brownian motion (like all Lévy processes) admits the Lebesgue measure as an invariant measure, hence, not finite.

4. A (squared) Bessel process exhibits the same property, namely an infinite invariant measure.

Proof. 1. For $\phi: \mathbb{R} \rightarrow \mathbb{R}$ in $C^{2}$, consider the Smoluchowski equation

$$
d X_{t}=\phi^{\prime}\left(X_{t}\right) d t+d W_{t},
$$

where $W_{t}$ is a standard Brownian motion. Then the measure $\mu(d x)=$ $e^{2 \phi(x)} d x$ is invariant for the process $\left(X_{t}\right)$. If we consider now an OrnsteinUhlenbeck process (with a standard deviation equal to 1 for implicity), then $d X_{t}=\left(a-b X_{t}\right) d t+d W_{t}, a, b>0, \phi^{\prime}(x)=a-b x, \phi(x)=c+a x-$ $\frac{b x^{2}}{2}$, and $\mu(d x)=e^{-b x^{2}+2 a x+c} d x$ is an invariant measure (normalized to 1 through $c$ ), and we recognize the Gaussian density. In the general case of an Ornstein Uhlenbeck process reverting to the mean $m$, and with a standard deviation equal to $\sigma$, the invariant measure will be $\mathcal{N}\left(m, \sigma^{2}\right)$.

2. We recall the definition of the squared-root (or CIR) process introduced in finance by Cox, Ingersoll, and Ross [3]:

$$
d X_{t}=\left(\delta-b X_{t}\right) d t+\sigma \sqrt{X_{t}} d W_{t} .
$$

We remember that a CIR process is the square of the norm of a $\delta$ dimensional Ornstein-Uhlenbeck process, where $\delta$ is the drift of the CIR process at 0 . The semi-group of a CIR process is the radial projection of the semi-group of an Ornstein Uhlenbeck. If we note $v$, the image of $\mu$ by the radial projection

$$
\int v(d r) \phi(r)=\int \mu(d x) P_{t}(\phi|\cdot|)(x)=\int \mu(d x) P_{t} \phi(|x|)=\int v(d r) P_{t} \phi(r) .
$$

Hence $v$, image of $\mu$ by the norm application, is invariant for $P_{t}$.

3. Consider a Levy process $\left(\mathcal{L}_{t}\right)$ and $P_{t}$ its semi-group. Then

$$
\begin{gathered}
P_{t}(x, f)=E_{0}\left[f\left(x+\mathcal{L}_{t}\right)\right] \\
\int d x P_{t}(x, f)={ }_{\text {Fubini }} E_{0}\left[\int f\left(x+\mathcal{L}_{t}\right) d x\right]=\int f(y) d y .
\end{gathered}
$$

Consequently, the Lebesque measure is invariant for the process $\left(\mathcal{L}_{t}\right)$.

4. We use the well-known relationship between a Bessel process and the norm of Brownian motion and also obtain an infinite invariant measure for Bessel processes. 
Having covered the fundamental types of Markovian diffusions used in finance, we are led to propose the following definition.

Definition 2. Given a Markov diffusion $\left(X_{t}\right)$, we say that the process $\left(X_{t}\right)$ exhibits mean reversion if and only if it admits a finite invariant measure.

\section{Remarks.}

1. The definition does not necessarily involve the drift of a stochastic differential equation satisfied by $\left(X_{t}\right)$, as also suggested in [11].

2. It allows inclusion of high-dimensional non-Markovian processes driving energy commodity prices or volatility levels.

3. Following [13], we can define the set

$$
T_{P}=\left\{\text { probability measure } \mu \text { such that } \mu P_{t}=\mu \forall t \geq 0\right\} .
$$

Then the set $T_{P}$ is convex and closed for the tight convergence topology (through Feller's property) and possibly empty. If $T_{P}$ is not the empty set and compact (for the tight convergence topology), there is at least one extremal probability $\mu^{*}$ in $T_{P}$. Then the process $\left(X_{t}\right)$ is ergodic for this measure $\mu^{*}$ : for any set $A \in \mathcal{F}_{\infty}^{X}$ that is invariant by the time translators $\left(\theta_{t}\right)_{t \geq 0}$, then $P_{\mu^{*}}(A)=0$ or 1 , where we classically denote $\mathcal{F}_{\infty}^{X}$ the natural filtration of the process $\left(X_{t}\right)$. The time-translation operator $\theta_{t}$ is defined on the space $\Omega$ by $\left(\theta_{t}(X)\right)_{s}=X_{t+s}$. It follows by Birkhoff's theorem that, for any function $F \in L^{1}\left(P_{\mu^{*}}\right)$,

$$
\frac{1}{t} \int_{0}^{t} F\left(X_{s}\right) d s \rightarrow E_{P_{\mu^{*}}}[F(X)] \quad P_{\mu^{*}}-\text { a.s. }
$$

when $t \rightarrow \infty$. The interpretation of this result is the following: the longrun time average of a bounded function of the ergodic process $\left(X_{t}\right)$ is close to its statistical average with respect to its invariant distribution. This property is crucial in finance, as the former quantity is the only one we can hope to compute using an historical database of the process $\left(X_{t}\right)$.

\section{An Econometric Approach to Mean Reversion in Energy Commodity Prices}

We recall the classical steps in testing mean reversion in a series of prices $\left(X_{t}\right)$. The objective is to check whether in the representation

$$
X_{t+1}=\rho X_{t}+\varepsilon_{t},
$$

the coefficient $\rho$ is significantly different from 1 . The $\mathcal{H}_{0}$ hypothesis is the existence of a unit root (i.e., $\rho=1$ ). A $p$-value smaller than 0.05 allows one to reject the $\mathcal{H}_{0}$ hypothesis with a confidence level higher than 0.95 , in which case the process is of the mean-reverting type. Otherwise, a unit root is uncovered, and the process is of the "random walk" type. The higher the $p$-value, the more the random walk model is validated. 


\subsection{Mean-Reversion Tests}

They are fundamentally of two types:

(i) The Augmented Dickey-Fuller (ADF) consists in estimating the regression coefficient of $p(t)$ on $p(t-1)$. If this coefficient is significantly below 1 , it means that the process is mean reverting; if it is close to 1 , the process is a random walk.

(ii) The Phillips-Perron test consists in searching for a unit root in the equation linking $X_{t}$ and $X_{t+1}$. Again, a high $p$-value reinforces the hypothesis of a unit root.

\subsection{Statistical Properties Observed on Oil and Natural Gas Prices}

For crude oil,

- a mean-reversion pattern prevails over the period 1994-2000;

- it changes into a random walk (arithmetic Brownian motion) as of 2000;

whereas for natural gas,

- there is a mean-reversion pattern until 1999;

- since 2000, a change into a random walk occurs, but with a lag compared to oil prices;

- during both periods, seasonality of gas prices tends to blur the signals.

For US natural gas prices over the period January 1994-October 2004, spot prices are proxied by the New York Mercantile Exchange (NYMEX) one-month futures contract. Over the entire period Jan 1994-Oct 2004, the ADF $p$-value is 0.712 and the Phillips Perron $p$-value is 0.1402 , whereas over the period Jan 1999-Oct 2004, the ADF $p$-value is 0.3567 and the Phillips Perron $p$-value is 0.3899 . Taking instead log-prices, the numbers become

\begin{tabular}{|l|r|}
\hline Jan 94 - Oct 04 & Jan 99 - Oct 04 \\
\hline ADF $p$-value $=0.0863$ & ADF $p$-value $=0.4452$ \\
\hline Phillips Perron $p$-value $=0.0888$ & Phillips Perron $p$-value $=0.4498$ \\
\hline
\end{tabular}

Over the last five years of the period, the arithmetic Brownian motion assumption clearly prevails and mean reversion seems to have receded.

For West Texas Intermediate (WTI) oil spot prices over the same period January 1994-October 2004, again spot prices are proxied by NYMEX onemonth futures prices, and the tests are conducted for log-prices.

\begin{tabular}{|l|c|}
\hline $1994-2004$ & Jan 1999 - Oct 2004 \\
\hline ADF $p$-value 0.651 & ADF $p$-value 0.7196 \\
\hline Phillips Perron 0.5048 & Phillips Perron 0.5641 \\
\hline
\end{tabular}

The mean-reversion assumption is strongly rejected over the whole period and even more so over the recent one. Because of absence of seasonality, the behavior of a random walk is more pronounced in the case of oil log-prices than in the case of natural gas. 


\section{The Economic Literature on Mean Reversion in Commodity Prices}

In [1], the term structure of futures prices is tested over the period January 1982 to December 1991, for which mean reversion is found in the 11 markets examined, and it is also concluded that the magnitude of mean reversion is large for agricultural commodities and crude oil, and substantially less for metals. Rather than examining evidence of ex-post reversion using time series of asset prices, [1] uses price data from futures contracts with various horizons to test whether investors expect prices to revert. The "price discovery" element in forward and futures prices is related to the famous "Rational Expectations Hypothesis" long tested by economics, for interest rates in particular (cf. [9]), and stating that forward rates are unbiased predictors of future spot rates. [1] analyzes the relation between price levels and the slope of the futures term structure defined by the difference between a long maturity future contract and the first nearby. Assuming that futures prices are unbiased expectations (under the real probability measure) of future spot prices, an inverse relation between prices and this slope constitutes evidence that investors expect mean reversion in spot prices, as it implies a lower expected future spot when prices rise. The authors conclude the existence of mean reversion for oil prices over the period 1982-1999; however, the same computations conducted over the period 2000-2005 leads to inconclusive results.

Pindyck [12] analyzes 127 years (1870-1996) of data on crude oil and bituminous coal, obtained from the US Department of Commerce. Using a unit root test, he shows that prices mean revert to stochastically fluctuating trend lines that represent long-run total marginal costs but are themselves unobservable. He also finds that during the time period of analysis, the random walk distribution for log-prices, i.e., the geometric Brownian motion for spot prices, is a much better approximation for coal and gas than oil. As suggested by Figure 4, the recent period (2000-2006) has been quite different.

One way to reconcile the findings in [12] and the properties we observed in the recent period described in this paper, is to "mix" mean reversion for the spot price towards a long-term value of oil prices driven by a geometric Brownian motion. The following three-state variable model also incorporates stochastic volatility:

$$
\begin{aligned}
d S_{t} & =a\left(L_{t}-S_{t}\right) S_{t} d t+\sigma_{t} S_{t} d W_{t}^{1}, \\
d y_{t} & =\alpha\left(b-y_{t}\right) d t+\eta \sqrt{y_{t}} d W_{t}^{2}, \quad \text { where } y_{t}=\sigma_{t}^{2}, \\
d L_{t} & =\mu L_{t} d t+\xi L_{t} d W_{t}^{3},
\end{aligned}
$$

where the Brownian motions are positively correlated. The positive correlation between $W^{1}$ and $W^{2}$ accounts for the "inverse leverage" effect that prevails for commodity prices (in contrast to the "leverage effect" observed in the equity markets), whereas the positive correlation between $W^{1}$ and $W^{3}$ translates the fact that news of depleted reserves will generate a rise in both spot and long-term oil prices. 


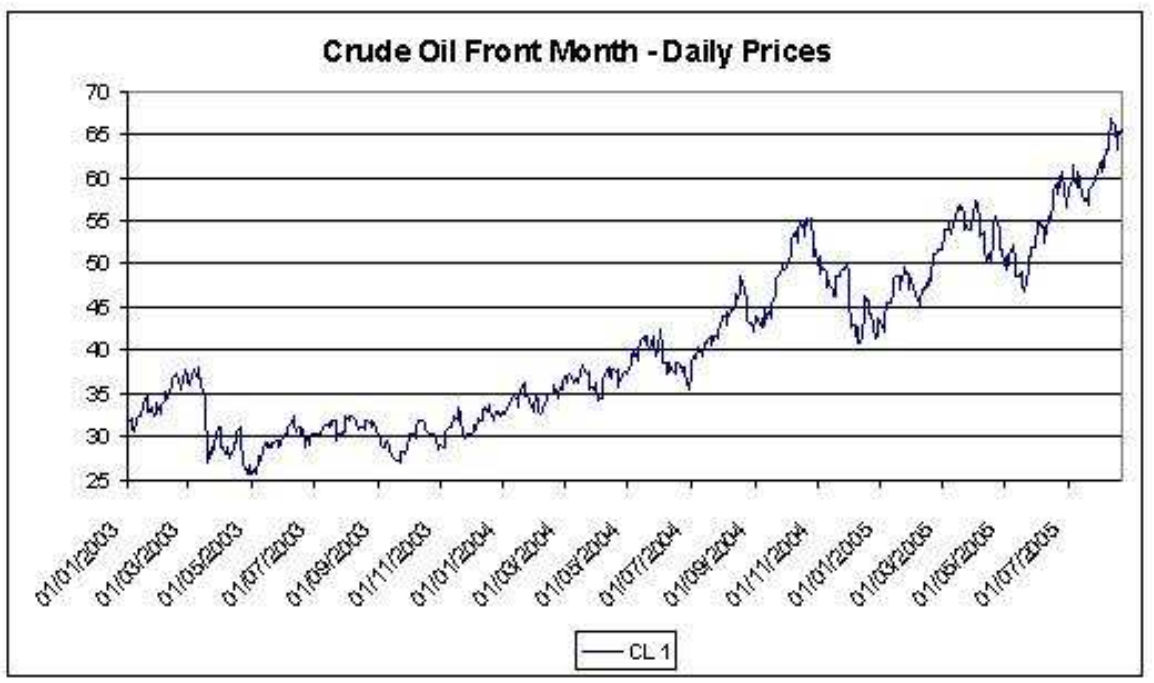

Fig. 4. NYMEX Crude Oil Front: month daily prices over the period Jan 2003-Aug 2005.

\section{Conclusion}

From the methodological standpoint, more work remains to be done in order to analyze in a unified setting (i) the mathematical properties of existence of an invariant measure and ergodicity for a stochastic process, and (ii) the meanreversion behavior as it is intuitively perceived in the field of finance. From an economic standpoint, the modeling of oil and natural gas prices should incorporate the recent perception by market participants of the importance of reserves uncertainty and exhaustion of these reserves.

\section{References}

1. H. Bessembinder, J. Coughenour, P. Seguin and M. Smoller. Mean reversion in equilibrium asset prices: Evidence from the futures term structure. Journal of Finance, 50:361-375, 1995.

2. F. Black and M. Scholes. The pricing of options and corporate liabilities. Journal of Political Economy, 81:637-659, 1973.

3. J.C. Cox, J.E. Ingersoll and S.A. Ross. A theory of the term structure of interest rates. Econometrica, 53:385-408, 1985.

4. A. Eydeland, and H. Geman. Pricing power derivatives. RISK, 71-73, September 1998.

5. J.P. Fouque, G. Papanicoleou and K.R. Sircar. Derivatives in Financial Markets with Stochastic Volatility. Cambridge University Press, 2000. 
6. H. Geman. Commodities and Commodity Derivatives: Modeling and Pricing for Agriculturals, Metals and Energy. Wiley, 2005.

7. R. Gibson and E.S. Schwartz. Stochastic convenience yield and the pricing of oil contingent claims. Journal of Finance, 45:959-976, 1990.

8. R.Z. Has'minskii. Stochastic Stability of Differential Equations. Sitjthoff \& Noordhoff, 1980.

9. Keynes, J.M. The Applied Theory of Money. Macmillan \& Co., 1930.

10. R.H. Litzenberger and N. Rabinowitz. Backwardation in oil futures markets: Theory and empirical evidence. Journal of Finance, 50(5):1517-1545, 1995.

11. M. Musiela. Some issues on asset price modeling. Isaac Newton Institute Workshop on Mathematical Finance, 2005.

12. R. Pindyck. The dynamics of commodity spot and futures markets: A primer. Energy Journal, 22(3):1-29, 2001.

13. G. Pages. Sur quelques algorithmes recursifs pour les probabilites numériques. ESAIM, Probability and Statistics, 5:141-170, 2001.

14. E.S. Schwartz. The stochastic behavior of commodity prices: Implications for valuation and hedging. Journal of Finance, 52(3):923-973, 1997. 